\title{
Causas que intervienen en la motivación del alumno en la enseñanza-aprendizaje de idiomas: el pensamiento del profesor
}

\author{
Nieves RODRÍGUEZ-PÉREZ \\ Universidad de Oviedo \\ Anglogermánica y Francesa \\ nirope@uniovi.es
}

Recibido: enero 2011

Aceptado: marzo 2012

\section{RESUMEN}

En este artículo presentamos las opiniones de profesores de distintos ámbitos educativos acerca de las causas personales, académicas y metodológicas que intervienen en la motivación del alumno en el proceso de enseñanza-aprendizaje de idiomas.

Partimos de la hipótesis de que la aprehensión de una nueva lengua-cultura genera en el aprendiz movimientos emotivos específicos que influyen en la motivación del estudiante por el aprendizaje de un idioma y que el interés de los docentes por esos factores y el conocimiento que tengan de sus modos operativos pueden mejorar el proceso de enseñanzaaprendizaje.

Los resultados avalan el importante papel que juegan los contextos en los que está inmerso el alumno en el desarrollo de la motivación tanto el contexto sociocultural -familiar, de amistades - como el entorno del aula -el grupo de alumnos y especialmente el profesor, su actitud, su estilo personal de enseñanza, sus estrategias para promover la interacción y su empeño por fomentar la empatía entre él y el grupo de alumnos pudiendo modificar los intereses, las necesidades e incluso las habilidades del individuo -. De igual manera, verifican que el aprendizaje de otra lengua-cultura requiere la participación del alumno en las actividades de clase, poniendo en riesgo su ego lingüístico, por ejemplo, ante la posible comisión de errores, afectando a su motivación. Además, supone el reconocimiento de otras formas de pensamientos lo que requiere del aprendiz la modificación de esquemas adquiridos así como la aprehensión de otros.

Palabras clave: motivación, emoción, actitud, interculturalidad, autoestima.

Causes intervening in pupil's motivation for language learning: the teacher's thought.

\begin{abstract}
This article shows the opinion of teachers from several educational scopes on the personal, academic and methodological causes which intervene in pupil's motivation within the language teaching-learning process.

The starting hypothesis is that the apprehension originated by a new language-culture generates certain emotional movements on the learner which influence the motivation the
\end{abstract}


students have on learning a language. Also the interest shown by the teacher on such factors as well as the knowledge on their operating ways may improve the teaching-learning process.

The results guarantee the important role played by the contexts where the learner is immersed when developing the motivation both the social and cultural environment family, friends- as well as the classroom context -the group of students and particularly the teacher's attitude, personal teaching style, strategies to promote interaction and determination to promote empathy between him and the group of students, being able to modify the interests, needs or even individual's skill-. In a similar way, they verify that learning another language-culture requires the student's participation in the classroom activities, putting their linguistic ego at risk, i.e. facing the possibility of making mistakes which may affect their motivation. Furthermore, it involves the recognition of other ways of thought, which requires the learner to modify acquired schemes and to apprehend others.

Key words: motivation, emotion, attitude, interculturality, self-esteem.

\section{Les causes qui interviennent dans la motivation de l'élève dans l'enseignement-apprentissage de langues étrangères : la pensée du professeur}

\section{RÉSUMÉ}

Dans cet article nous présentons les avis de professeurs de différents domaines éducatifs sur les causes personnelles, académiques et méthodologiques qui interviennent dans la motivation de l'élève dans le processus d'enseignement-apprentissage de langues étrangères. Nous partons de l'hypothèse que l'appréhension d'une nouvelle langue-culture produit chez l'apprenti des mouvements émotifs spécifiques qui influencent la motivation de l'étudiant pour l'apprentissage d'une langue et que l'intérêt des enseignants pour ces facteurs ainsi que leurs connaissances en ce qui concerne leurs manières opérationnelles peuvent améliorer le processus d'enseignement-apprentissage.

Les résultats garantissent le rôle important que jouent les contextes socioculturel - parent, amis- et la salle de classe - le groupe d'élèves et spécialement le professeur, son attitude, son style personnel d'enseignement, ses stratégies pour promouvoir l'interaction et sa persistance pour favoriser l'empathie entre lui et le groupe d'élèves en pouvant modifier les intérêts, les besoins et y compris les habilités de l'individu- pour le développement de la motivation. De la même manière, ceux-ci vérifient que l'apprentissage d'une autre langueculture requiert la participation de l'élève dans les activités de classe, en mettant en risque son ego linguistique, par exemple, devant la possible commission d'erreurs, en affectant sa motivation. En outre, cela suppose la reconnaissance d'autres formes de pensées ce qui requiert de l'apprenti la modification de schémas acquis ainsi que l'appréhension des autres.

Mots clé : Motivation, émotion, attitude, interculturalité, auto-estime

SUMARIO: Introducción. Material y métodos. Presentación de los resultados. A qué causas personales, académicas y metodológicas adjudica usted la motivación de los alumnos? Análisis pormenorizado de cada categoría. A qué causas personales, académicas y metodológicas adjudica usted la desmotivación de los alumnos? Análisis pormenorizado 
de cada categoría. Discusión de los resultados y conclusiones. Reflexión final. Referencias bibliográficas

\section{INTRODUCCIÓN}

Las investigaciones sobre motivación y emoción en la adquisición de lenguas son un tema de preocupación creciente como constatan los numerosos textos surgidos en la última década. En la bibliografía especializada encontramos numerosas definiciones ${ }^{1}$ del concepto de motivación. Aunque en la actualidad ninguna de ellas se considera totalmente satisfactoria - se enuncian desde diferentes perspectivas: psicología, pedagogía, didáctica de idiomas ${ }^{2}$, etc.observamos que, a pesar de las diferencias terminológicas y conceptuales, las discrepancias fundamentales no son tan grandes. La mayoría de ellas emplean términos como impulsos, incentivos, estímulos, deseos, necesidades o intereses, estados o procesos internos que interactúan con factores externos pudiendo ser inducidos por ellos. En general, la conceptualizan como un proceso dinámico, cambiante, que genera, mantiene y dirige una conducta orientada hacia una meta, ya que si ésta no existe, no existe conducta motivada.

Así mismo, numerosas investigaciones aluden a una serie de variables que influyen en dicho proceso, las expectativas, los patrones atribucionales, el autoconcepto, los reforzadores, las metas, las emociones... Por consiguiente, este constructo tan amplio no se puede entender sin una interrelación de diversas dimensiones: una dimensión cognitiva; una dimensión contextual y una dimensión emotiva-afectiva.

En nuestro recorrido por la literatura especializada de enseñanza-aprendizaje de idiomas, observamos que numerosos autores otorgan al aprendizaje de una lengua extranjera características especiales. El alumno no solo debe adquirir nueva información sino también elementos simbólicos de la otra comunidad etnolingüística. El lenguaje es el canal de comunicación social, un sistema de códigos unidos a la identidad del sujeto, por lo que la adquisición de una nueva lengua produce efectos sobre la personalidad del aprendiz (Dörnyei, 1998; Williams y Burden, 1999; Pavlenko, 2002; Arnold, 2000). Y es que enfrentarse al estudio de una realidad lingüístico-cultural distinta a la materna moviliza en el

\footnotetext{
${ }^{1}$ Ames y Ames $(1984,1985,1989)$ presentan un excelente resumen de la gama de enfoques de la motivación. Kleinginna y Kleinginna (1981) han recopilado cien definiciones diferentes de motivación.

${ }^{2}$ Desde el ámbito de enseñanza aprendizaje de idiomas algunos autores expresan la dificultad que conlleva encontrar una definición: This difficulty in defining motivation is revealed, on the one hand, by the plethora of definitions of motivation, and on the other, by the abundance of theories of motivation which are associated with different psychological perspectives on human behaviour (p.e.: Manolopoulou-Sergi, 2004, 428).
} 
sujeto emociones positivas o negativas vinculadas al propio objeto de estudio, la lengua-cultura extranjera.

El contacto con otra realidad lingüístico-cultural requiere del aprendiz la modificación de esquemas adquiridos así como la adquisición de otros. Por una parte, la aprehensión de aspectos fónicos distintos de los de la lengua materna otra forma de pronunciación, de ritmo, de entonación, etc. - compromete al cuerpo del aprendiz. Por otra, la confrontación con formas de pensamiento y modos culturales distintos inducen a cuestionar los propios o a rechazar los ajenos. El alumno, cuando se enfrenta por primera vez al aprendizaje de un idioma, no parte como una tábula rasa, ya posee esquemas de comportamiento mental y social anclados en su contexto nativo, frecuentemente inconscientes y, por lo tanto, fuera de su control, que dificultan el reconocimiento de otros sistemas de pensamiento y cultura. Pero también, en algunos casos, el idioma extranjero puede ser el medio para expresar opiniones, sentimientos, deseos inhibidos por la propia lenguacultura. El estudio de un idioma puede hacer emerger vivencias negativas o positivas ocasionando respuestas condicionadas. Por ejemplo, un estudiante con experiencias negativas en el contacto con personas extranjeras manifiesta en general una mayor desmotivación y aversión al aprendizaje de un idioma que otro sujeto con experiencias positivas en las relaciones con extranjeros. Quizás se deba como relatan algunos autores a que apropiarse de una segunda lengua implica:

(...) una reclasificación de nosotros mismos y de todo lo que nos rodea, lo que nos convoca a una reconstrucción o reorganización de nuestra conciencia, $\mathrm{y}$, en último término, a una reconversión comportamental, a una reorientación de nuestro proceso de hominización que cuestiona el proceso realizado hasta ese momento en el marco de las interacciones mediadas por la lengua materna y por, si así fuese el caso, las otras lenguas segundas aprendidas (p.e.: Atienza et al, 2005, 38).

Durante el proceso de aprendizaje de un idioma, al alumno no sólo se le pide un esfuerzo cognitivo en términos de vocabulario, gramática y sintaxis, sino algo mucho más considerable: la necesidad de volver a categorizar la información, lo que conduce inevitablemente a una exigencia de conceptualización y, en última instancia, de experimentación de los acontecimientos internos o externos a nosotros (p.e.: Guiora, 1984, 8).

Sería un error pensar que los mecanismos de la motivación y de la emoción actúan en todos los aprendices por igual, pues los individuos difieren en sus intereses, motivos y cualidades emocionales. Por tanto, en el contexto del aula, el enseñante interesado por la incidencia de estos dos factores en los procesos de aprendizaje tendrá que tener en cuenta las diferencias individuales.

Ese espacio artificial, en el que entran en juego diferentes sujetos -el profesor, los aprendices y el grupo- se caracteriza por ser un gran potencial generador de emociones. Es algo que los profesores sabemos bien y de ahí surge nuestra 
preocupación por el diseño de materiales que logren mantener o fomentar en los alumnos la motivación-emoción ${ }^{3}$.

Partimos de la hipótesis de que en la actualidad existen cambios en la motivación de los alumnos y profesores ocasionados por las comunicaciones interculturales, necesidades profesionales o avances en las tecnologías de la comunicación. Y de que la adquisición de una nueva lengua genera emociones específicas susceptibles de potenciar o bloquear los procesos de aprendizaje. Esas emociones están vinculadas al hecho de que un idioma extranjero exige del aprendiz conductas fónicas, gestuales, comportamientos culturales y hábitos de pensamiento que cuestionan las de su propia lengua materna, afectando a su identidad. Creemos que la experiencia de los profesores puede aportar nuevas ideas que ayuden a descifrar este constructo tan amplio.

En definitiva, el objetivo del estudio ha sido conocer las creencias de los docentes sobre las causas personales, académicas o metodológicas que intervienen en el proceso de enseñanza-aprendizaje de una lengua extranjera, y cómo son esas creencias. Estos resultados nos pueden ayudar a actuar de manera más adecuada en cada contexto.

\section{MATERIAL Y MÉTODOS}

Existen diferentes alternativas que nos permiten conocer el pensamiento de los profesores acerca de lo que hace o ha hecho, cree o siente; sobre los factores que intervienen en la motivación del alumno y su influencia en el proceso de enseñanza-aprendizaje de idiomas. En nuestro caso optamos por un enfoque biográfico, por lo que hemos de contar como relatan Bolívar et al. (p.e.: 2001, 147), con al menos cuatro elementos: Un narrador, que cuenta sus vivencias; un intérprete o investigador, que elabora el informe de los relatos; textos donde se recoge lo que es narrado y por último lectores a los que se dirige el informe.

En consecuencia, procedimos al diseño de una encuesta con preguntas de respuesta abierta que posibilite la obtención de significados de las dimensiones afectivas, cognitivas, sin desdeñar, naturalmente, aquellas informaciones de carácter generalizable o cuantificable que podamos recabar tras los diversos análisis cualitativos y cuantitativos de las respuestas obtenidas, aunque esta perspectiva es meramente orientativa puesto que nuestro estudio se ha realizado desde un enfoque fundamentalmente cualitativo.

Para la validación del cuestionario realizamos un "Pretest" y lo aplicamos a 12 profesores expertos, escogidos de tal forma que representaran la diversidad de sujetos a los que iba a ser dirigido, procedentes de las diferentes etapas educativas en las que se centraría nuestra investigación (Primaria, Secundaria y Universidad).

\footnotetext{
${ }^{3} \mathrm{Si}$ unimos así ambos términos es porque creemos que los enseñantes suelen identificar ambos conceptos, aunque prefieran hablar de motivación.
} 


\section{Definición y selección de la muestra a encuestar}

Puesto que nuestro estudio se enmarca dentro de las investigaciones cualitativas, su interés no reside en la cantidad de sujetos encuestados sino en la exhaustividad, la intensidad y profundidad de las observaciones. Por ello, la muestra incluye una selección de participantes con un perfil determinado idóneo para responder consecuentemente el cuestionario. Así pues, contactamos con profesores que se encuentran inmersos en preocupaciones específicas vinculadas con el aprendizaje y enseñanza de un idioma, por ejemplo que hubieran realizado cursos de formación y actualización relacionados con la enseñanza-aprendizaje de idiomas. Además, también nos interesaba conocer las estrategias docentes en los tres niveles educativos, por consiguiente, seleccionamos una muestra de profesores que imparten docencia en Primaria, Secundaria y Universidad; de alemán, francés e ingles y con diferentes años de experiencia: noveles, entre diez y veinte años y con más de veinte años de práctica. De los 90 profesores con los que se contactó, recibimos 58 cuestionarios cumplimentados.

\section{Procesamiento de los datos}

El manejo y tratamiento de la ingente cantidad de información y su interrelación con los diferentes encuestados se realizó mediante el programa informático Atlas/ti 5 versión 3.03. Para el procesamiento de los datos codificamos y establecimos una serie de categorías que se fueron afinando y modificando sucesivamente a medida que se avanzaba en el análisis. De esta manera se establece una interacción continua entre la recogida de datos que contienen las encuestas y el análisis que se va realizando. Dicho proceso, como especifican diversos autores - Gil, García y Rodríguez (1995), Colás Bravo y Buendía Eisman (1994), LeCompte (2000) - se realiza en varias fases: la lectura inicial de los datos, codificación y categorización y la obtención de conclusiones. Este proceso supuso una constante revisión, relectura de las encuestas, adecuación de los códigos a medida que se progresaba en su estudio y valoración.

\section{PRESENTACIÓN DE LOS RESULTADOS}

3.1. Pregunta $n^{0}$ 1: ¿A qué causas personales, académicas, metodológicas adjudica usted la motivación de sus alumnos?

Prácticamente el cincuenta por ciento de los docentes adjudica al entorno familiar y cultural del alumno la responsabilidad de su motivación. En relación a las causas académicas el factor más importante es el expediente académico y en lo que respecta a las causas metodológicas destaca la influencia del enfoque comunicativo. Sus respuestas se pormenorizan en la siguiente tabla: 


\begin{tabular}{|l|c|}
\hline Categorías y Subcategorías & Número Profesores \\
\hline 1. Causas personales & \\
\hline Entorno familiar-sociocultural de alumno & 32 \\
\hline Edad alumno & 3 \\
\hline Capacidad y habilidad para la adquisición del idioma & 9 \\
\hline Autoestima del alumno & 5 \\
\hline Actitud del profesor en el aula & 19 \\
\hline Implicación del alumno en el proceso de aprendizaje & 6 \\
\hline Motivación profesor & 9 \\
\hline Aprendizaje del idioma por motivos profesionales & 15 \\
\hline El idioma posibilita la comunicación con otras & 8 \\
\hline culturas & 2 \\
\hline Estado fisico de estudiante & \\
\hline 2. Causas académicas & 12 \\
\hline Obtención de un buen expediente académico & 9 \\
\hline Experiencias anteriores & 5 \\
\hline Tamaño del grupo & 2 \\
\hline Homogeneidad de conocimientos en el grupo & 3 \\
\hline Dotación de material en las aulas & 3 \\
\hline Programación: metas y criterios evaluación & 6 \\
\hline Causas generadas por el centro educativo & 10 \\
\hline 3. Causas metodológicas & \\
\hline Metodologías atractivas & 20 \\
\hline Técnicas de trabajo que facilitan el aprendizaje & 6 \\
\hline Adaptación materiales a las necesidades individuales & \\
\hline Dificultad de la tarea & \\
\hline
\end{tabular}

Tabla 1. Categorías, subcategorías y número de profesores que aluden a cada una de ellas. 


\subsubsection{Análisis pormenorizado de cada categoría Causas personales}

\section{Entorno familiar y sociocultural del alumno}

El contexto socio-cultural del que procede el alumno, las circunstancias familiares (P20) ${ }^{4}$ o la extracción social (P22) constituye una de las causas principales de la motivación o desmotivación del estudiante hacia el aprendizaje de idiomas. Los informantes afirman la existencia de una relación directa entre los intereses y actitudes familiares hacia el aprendizaje de la lengua meta y la conducta del individuo, que reproduce e imita sus estimaciones y valores: Las familias valoran mucho estos aprendizajes $y$, aunque no lo parezca, los niños y niñas reflejan las actitudes y valores familiares (P10). También aluden al ambiente afectivo (P25) y a las buenas relaciones en su entorno familiar y social (P31) como generadores de la motivación. Los profesores opinan que los padres han de implicarse en la ejecución de las tareas de sus hijos y no limitarse únicamente a poner a su disposición el material necesario para alcanzar las metas de aprendizaje: Nunca estuvieron las casas tan llenas de todo (libros, ordenadores...) y nunca se estudió menos (P55). Esta implicación conlleva, como apunta un profesor del ámbito educativo de Primaria, que los padres les inculquen desde edades tempranas la importancia del conocimiento de una lengua extranjera (P7).

Así mismo, desde el entorno familiar, se ha de incentivar en el estudiante el interés por el contacto con el país de la lengua extranjera. Las reflexiones de los profesores parten de sus observaciones a las familias que se han preocupado porque sus hijos se beneficien de becas, intercambios, de que realicen viajes culturales, clases de idiomas, salidas al extranjero (P13).

\section{Edad del alumno}

Algunos docentes opinan que la decisión de que los alumnos comiencen el estudio de un idioma en edades tempranas depende únicamente del interés familiar y de las ofertas del centro educativo. Unos docentes, de la etapa educativa de Primaria (P17), manifiestan que la motivación es mayor en los alumnos que comienzan a estudiar idiomas en edades infantiles.

En esta subcategoría incluimos también a los enseñantes que se refieren a la etapa de la adolescencia de los estudiantes como una época de pasotismo y de desinterés por el aprendizaje en general.

${ }^{4}$ Identificamos a los autores de los textos con la letra $\mathrm{P}$ para mantener el anonimato de los profesores. El número se refiere al puesto que ocupan los docentes en el programa informático ATLAS/ti. 


\section{Capacidad y habilidad del alumno para la adquisición del idioma}

Algunos profesores aseguran la existencia de habilidades específicas para el aprendizaje de idiomas en determinados alumnos. Detallan que los estudiantes que presentan dificultades en el aprendizaje general (P12) no se sienten capaces de aprender una lengua extranjera, se desmotivan e incluso pierden sus hábitos de estudio (P8).

\section{Autoestima del alumno}

Otro de los temas, relacionado con el anterior, es la seguridad del aprendiz por alcanzar las metas propuestas:

Un alumno está motivado cuando está seguro de sí mismo (...). Por tanto, hay un proceso adecuado en el aprendizaje y un aumento de su autoestima por el trabajo bien hecho (P1).

Además, la realización exitosa de las actividades propuestas potencia la confianza del aprendiz y disminuye el rechazo a realizar otras nuevas: La confianza en que todos pueden llegar al final (P2).

\section{Actitud del profesor en el aula}

Varios relatos aluden a los lazos afectivos existentes entre profesor-alumnogrupo de alumnos y relacionan la motivación con el grado de confianza, familiaridad y compañerismo - A las relaciones que mantienen tanto con su profesor, como con sus compañeros (P15)- Si el clima en el contexto de aprendizaje y la relación establecida entre el grupo y el enseñante son valorados positivamente por el aprendiz, circunstancias como la comisión de errores o el fracaso en la realización de tareas no afectarán ni a la autoestima del alumno ni a su motivación. Para construir un clima motivador en el aula, resulta necesario, como afirma P24, emplear un tono de voz y maneras respetuosas para dirigirse a los alumnos, escucharlos y permitirles que se expresen con libertad, siempre desde la perspectiva de respeto hacia el profesor y hacia sus compañeros.

En la misma línea argumental se expresa otro docente: El alumno debe ver al profesor como una persona mayor, con autoridad pero, al mimo tiempo, accesible, cercano, risueño, alegre... (P34). Los encuestados emplean diversas estrategias para promover la interacción entre los miembros del grupo y entre el alumnoprofesor como la memorización de los datos personales del aprendiz — nombre y lugar de procedencia - para poder dirigirse a ellos de forma individualizada dentro del grupo: se nota que les gusta dejar de ser parte de la "masa" (así es como ellos creen que el profesorado los ve) para recuperar su propio nombre (P29). Diversos informantes mencionan las ventajas de poseer un carácter extravertido y alegre y aluden a los efectos beneficiosos del empleo del humor por el enseñante: Pienso que el buen humor es necesario. El profesor enseña más con lo que es que con lo 
que dice ( $\mathrm{P} 2)$. Otro encuestado manifiesta: Un profesor amargado no debería estar en un aula $(\mathrm{P} 34)$.

Una docente de Universidad añade que la motivación del aprendiz se ve reforzada cuando el docente da muestras de su honestidad (por ejemplo, en cuanto a sus dudas lingüísticas); cuando no actúa ante el alumno como la profesora que lo sabe todo (P29), sino como alguien que aún desea y tiene que aprender.

\section{Motivación del profesor}

La motivación de los enseñantes, el interés o desinterés del profesor por la asignatura (P39) es imprescindible para generar un clima agradable en el aula que motive al alumno y al docente: Que el profesor trabaje en un clima favorable. Para motivar debe estar motivado (P28).

\section{Implicación del alumno en su proceso de aprendizaje}

Los encuestados destacan la importancia de responsabilizar e implicar al alumno en su aprendizaje, para lo cual, algunos informantes confieren al profesor una labor esencial:

Saber transmitir entusiasmo, admiración, placer por la lengua y su uso. Hacerles ver que las tareas que están realizando las realizan en su propio provecho, para que su desarrollo afectivo e intelectual sea efectivo, para que en el futuro sean ellos responsables de su propio vivir como personas (P46).

\section{Aprendizaje del idioma por motivos profesionales}

Varios encuestados adjudican las causas de la motivación al carácter práctico de los estudios, es decir, a la creencia de que el conocimiento de idiomas contribuirá y facilitará la inserción profesional del alumno: Perspectivas laborales y convicción de que tienen que trabajar en el futuro y necesitarían el inglés para ello (P35). Un profesor, incluso, le concede a esta circunstancia mayor influjo en la motivación del alumno que a la metodología que el docente aplica al aula: (...) el que el profesor se incline por una u otra metodología no implicará, necesariamente, que se sientan más o menos motivados por la asignatura ya que la motivación viene dada por la "utilidad" de los estudios que se realizan (P37).

Una profesora, del ámbito educativo de Secundaria, manifiesta taxativamente que si el alumno estima como irrelevante para su futuro profesional el dominio de un idioma, se produce un descenso en su motivación: Los alumnos consideran la LE una materia difícil y farragosa, con poca relevancia en su formación (P17).

\section{El idioma posibilita la comunicación y el conocimiento de otras culturas}

Los docentes apuntan al deseo de los alumnos de contactar con jóvenes de otros países, de entablar amistades y como una herramienta necesaria para viajar al 
extranjero (P48), puesto que, en palabras de $\mathrm{P} 35,(. .$.$) la comprobación de que la$ lengua (inglés) les sirve para comunicarse (P35).

Un profesor de inglés (P30) relaciona la motivación con el deseo de conocer e imitar las modas procedentes de la cultura inglesa. Los alumnos valoran muy positivamente que las referencias culturales del país de la lengua meta procedan de las experiencias del docente en el extranjero: Conocer los temas, los personajes, los patrones de moda te da una imagen positiva a los ojos de los alumnos. No eres el típico profesional que se pasa la vida entre libros y no comparte con ellos... (P29).

Cuatro enseñantes atribuyen el aumento de la motivación en el alumno a su actitud positiva ante el descubrimiento de otras formas de vida, a su deseo de superación, enriquecimiento personal, curiosidad e interés por ampliar los conocimientos hacia otros campos del saber y otras culturas.

\section{Estado fisico del estudiante}

La salud de los alumnos es otro factor esencial que destacan, especialmente, enseñantes de Primaria para quienes la motivación aumenta cuando descansan y se alimentan adecuadamente: buena salud, alimentación y descanso adecuados (P31).

\section{Causas académicas}

Obtención de un buen expediente académico y expectativas universitarias

Ocho profesores relacionan la motivación con la obtención de recompensas como la adquisición de un buen expediente que les da acceso a cualquier carrera universitaria. (P45). Sin embargo, algunos alumnos interpretan las recompensas como un "pago" por su esfuerzo (P23). No obstante, este tipo de premio, en opinión de P14, no debe ser la causa que motive al estudiante, sino el efecto de su motivación.

\section{Experiencias anteriores en el aprendizaje}

Directa o indirectamente se hace referencia a las experiencias anteriores en el aprendizaje. Unos docentes opinan que la motivación se genera en los primeros contactos con el idioma, si las experiencias fueron o no gratificantes (P11). Un profesor de Secundaria manifiesta que cuando el aprendiz llega al aula desmotivado la labor del docente resulta infructuosa: Los alumnos de ESO ya tienen experiencia de aprendizaje de una lengua extranjera y sus expectativas ya están creadas (P35).

Algunos informantes (P56, P26) aluden al nivel con el que los estudiantes llegan a un curso: el buen conocimiento puede motivar para seguir mejorando (P26). El docente P5 expresa que cuando el alumno llega al aula con un historial de fracaso escolar, carece de motivación para lograr resultados más positivos y cuando 
los alcanza los atribuye a la buena suerte. En la misma línea argumental, algunos profesores al referirse a las experiencias previas precisan que el bajo nivel lingüístico genera un sentimiento de inferioridad con respecto a los compañeros que les bloquea emocionalmente e imposibilita la adquisición de nuevas habilidades.

\section{Tamaño del grupo}

Algunos encuestados apuntan a la estructura del grupo y concluyen que cuando el número de alumnos es reducido la motivación es mayor: Últimamente los desdobles en la ESO favorecen las clases al haber reducido el número de alumnos (P2). Por el contrario, un número elevado de aprendices en el aula impide la realización de actividades cooperativas pensadas para el desarrollo de destrezas comunicativas: El inglés hay que practicarlo todos los días para soltarte a hablar. Con tantos alumnos muchas veces es imposible (P38).

Homogeneidad de conocimientos en el grupo

Dos profesores aluden directamente a la homogeneidad de conocimientos dentro del grupo: La motivación aumenta cuando el grupo es homogéneo y hay una mayoría de alumnos a los que les interesa la asignatura, esto fortalece al grupo (P1).

\section{Dotación de material en las aulas}

Los enseñantes expresan que las aulas bien dotadas, específicas para la impartición de idiomas, favorecen la motivación de los alumnos: dotación adecuada de material, aulas adecuadas y específicas, como aulas de informática, de música, laboratorios, gimnasios, de tecnología (P46).

\section{Programación: aclaración de las metas y criterios de evaluación}

Cuatro encuestados se refieren a la información recogida en las programaciones de las materias y advierten de que los alumnos han de estar informados tanto de las metas u objetivos que deben alcanzar, como de los criterios de evaluación. Otro profesor opina que los conocimientos prácticos de la lengua, las destrezas comunicativas, han de valorarse académicamente y no sólo tener en cuenta las puntuaciones obtenidas en el ejercicio escrito. Incluso como plantea un docente (P2) el alumno debe ser capaz de autoevaluarse y de conocer sus progresos

\section{Causas generadas por el centro educativo}

Entre las competencias de los centros educativos se encuentran la obligación de llevar a cabo la enseñanza-aprendizaje en el mejor de los escenarios posibles (...un ambiente hostil en el centro puede suponer un infierno, P16) y la de fomentar el conocimiento de lenguas extranjeras (Desde los centros educativos valoramos e insistimos en la importancia de manejar una L2, P10). Los responsables deben 
esforzarse en crear las condiciones más favorables, como (...) el establecimiento de un horario adecuado y conveniente ( $\mathrm{P} 31)$, o promover un clima de trabajo y cooperación entre los docentes y los departamentos (P16). Así mismo, reflexionan sobre la trascendencia de los intercambios con el extranjero, las becas, etc.

Los enseñantes apuntan a la coordinación entre los componentes del claustro para unificar criterios concernientes a la evaluación o a las metodologías de enseñanza: Siguen conviviendo diferentes concepciones de la enseñanza, diferentes métodos que desorientan a los alumnos, producen equivocos cuando se les propone trabajar de otro modo (P22).

Así mismo, P56 destaca que el centro de enseñanza debe intervenir en la distribución de los grupos y horarios para evitar que los estudiantes tengan cada año un profesor diferente.

\section{Causas metodológicas}

\section{Metodologías atractivas}

Numerosos testimonios se refieren al enfoque comunicativo como al modelo idóneo para generar la motivación, (...) alejado de las clases magistrales, en el que tienen que participar de forma activa, en grupo, haciendo uso de la lengua extranjera (P3). Un profesor declara que cuando el alumno logra superar el miedo a participar en las actividades de aula, se incrementa su motivación: La metodología comunicativa supone trabajar el "miedo al ridiculo" y a través de participación en clase, juegos y otras actividades van limando esas actitudes y favoreciendo la respuesta más madura $(\mathrm{P} 2)$.

Sin embargo, un informante se muestra escéptico ante el supuesto éxito de estas tareas en las que se desarrollan destrezas de tipo comunicativo: Sin embargo, el resultado muchas veces no es el esperado, porque la falta de fe en las tareas de comunicación encomendadas da paso a la dispersión... (P41).

Otro profesor alude positivamente a la competitividad entre los alumnos cuando trabajan en grupo: En mis clases he comprobado cómo su motivación aumenta cuando propongo actividades en equipos, especialmente con un pequeño grado de competitividad (P17). Opinión compartida por un número significativo de docentes que defienden las actividades grupales en el contexto del aula, al constatar que la competitividad intergrupal motiva al alumno a adquirir mejores resultados.

Técnicas de trabajo que faciliten el aprendizaje

Algunos encuestados afirman la conveniencia de que el alumno adquiera técnicas y estrategias de trabajo que pueda aplicar en otras disciplinas: Darles directrices que puedan reutilizar en otras asignaturas, por ejemplo, la estructura de la redacción desde el punto de vista del "plan cartésien" (P45). 
Adaptación de los materiales a las necesidades e intereses individuales

Encontramos varias alusiones a la práctica de una enseñanza en la que se fomente el aprendizaje autónomo. Para ello se requiere, la adaptación de materiales y métodos a las necesidades de los alumnos: En el plano metodológico, el eclecticismo, el uso de métodos y estrategias variadas y adaptadas a cada alumno y situación es otro elemento básico (P14), y, además, que las metodologías se adapten a los intereses, a la edad de los individuos (P26, P45). Por ello reclaman más personal de apoyo dentro del aula para ayudarles en el aprendizaje de forma individualizada $(\mathrm{P} 12)$.

En definitiva, la motivación está prácticamente garantizada si las tareas a realizar y la información que los alumnos reciben de su entorno son consideradas por éstos (...) útiles para alcanzar sus objetivos personales (P5). Algunos encuestados proponen, para captar la atención de los estudiantes y despertar su interés, actividades o comentarios basados en sus experiencias personales y/o en temas de actualidad, tendencias o personajes de moda (P30). Un docente de universidad expresa: Algún guiño contemporizador puede llevarse al aula con buenos resultados, especialmente si la propuesta es algo polémica: les anima a participar con su propia voz (P29).

En el lado opuesto, los encuestados atribuyen la desmotivación de los alumnos a diversos factores: a que los profesores no emplean herramientas nuevas para dinamizar la clase de idiomas; a los libros de texto, aburridos y con un vocabulario obsoleto, etc.: Cuando nos atenemos exclusivamente al manual y no permitimos una clase abierta (P44).

Grado de dificultad de la tarea

Un encuestado manifiesta que si los alumnos (...) tienen que estudiar más de lo que están dispuestos a hacer (P43) se genera un descenso de la motivación. Igualmente sucede si el grado de dificultad de la tarea es inferior al nivel del alumno: El nivel de exigencia ha sido casi siempre muy bajo. No es necesario esforzarse ni estudiar para aprobar, por lo tanto, ¿para qué hacer más? (P56).

A continuación nos referimos a las causas a las que los docentes atribuyen expresamente la desmotivación de los alumnos.

\subsection{Pregunta $\mathrm{n}^{0}$ 2: ¿A qué causas personales, académicas, metodológicas adjudica usted la desmotivación de sus alumnos?}

La causa personal más citada que origina la desmotivación de los alumnos es la vergüenza o el miedo a hablar en público, cometer errores, etc. La causa académica más alegada es el carácter obligatorio de la asignatura. No nos referimos a las causas metodológicas por estar citadas con anterioridad. 


\begin{tabular}{|l|c|}
\hline Categorías y Subcategorías & $\begin{array}{c}\text { Número } \\
\text { Profesores }\end{array}$ \\
\hline 1. Causas personales & 4 \\
\hline Miedo, vergüenza a intervenir en clase & 3 \\
\hline Procedencia del medio rural & 1 \\
\hline Influencia de los medios de comunicación & 2 \\
\hline Espíritu localista: estereotipos & \\
\hline 2. Causas académicas & 7 \\
\hline $\begin{array}{l}\text { Carácter obligatorio } \\
\text { Currículum sobrecargado de asignaturas y falta de } \\
\text { claridad en los objetivos }\end{array}$ & 4 \\
\hline Tabla 2. Categoris, subcategoras y número de profesores que auden a cada
\end{tabular}

Tabla 2. Categorías, subcategorías y número de profesores que aluden a cada una de ellas

\subsubsection{Análisis pormenorizado de cada categoría y subcategoría Causas personales}

Miedo, vergüenza a intervenir en clase: comisión de errores

Unos enseñantes se refieren al influjo en la motivación de sentimientos negativos como miedo al ridículo, inferioridad, timidez o vergüenza. Estos sentimientos surgen en el contexto de enseñanza y son generados por factores externos como la actitud del profesor o los propios compañeros. En el siguiente texto se relatan algunos abusos de los docente en la corrección de errores:

Los profesores sobrevaloramos la importancia de la corrección gramatical y no creamos situaciones que permitan valorar la variedad y viveza de la comunicación. Se abusa de los ejercicios escritos, se penalizan TODOS los errores, y creo que pocos profesores enseñan técnicas para el aprendizaje de la lengua y procedimientos eficaces de comunicación. Los alumnos consideran la LE una materia difícil y farragosa, con poca relevancia en su formación (P17).

Otros aluden a la relevancia concedida por algunos profesores a los aspectos de pronunciación y prosodia del idioma:

Un complejo de impotencia frente al aprendizaje de otras lenguas, en especial en el terreno de la fonética que es el gran caballo de batalla de los profesionales de la enseñanza de lenguas extranjeras en nuestro país; acompañando de ese otro complejo tan español como es el miedo al ridículo (P27). 
Los docentes manifiestan que la desaparición de estos sentimientos negativos favorece el interés por la "otra" lengua-cultura: Importantísimo la desaparición de ese filtro que les impide "dejarse llevar", no pasar "vergüenza" (P11).

\section{Procedencia del medio rural}

Los encuestados coinciden en señalar que en las zonas rurales los estudiantes están menos motivados que en las urbanas y lo justifican en que en este contexto no se ve la utilidad práctica del aprendizaje de una lengua extranjera. No obstante, algunos informantes constatan que el desarrollo de las comunicaciones está produciendo cambios evidentes en el mundo rural a favor del aumento en la motivación de los alumnos. A continuación presentamos el relato de una profesora que ha trabajado en ambos contextos:

Todo depende en gran parte del ambiente familiar y el entorno que les rodea. Personalmente he trabajado en un centro de una zona residencial con alto nivel de estudios de los padres y éstos influían mucho en los niños: libros en casa, viajes culturales, clases de idiomas, salidas al extranjero... La otra realidad la estoy viviendo en la zona rural donde los padres no tienen o no dedican tiempo a sus hijos, que crecen libremente ajenos a todo mundo exterior y en algunos casos te dicen convencidos que ellos para trabajar en el pueblo no van a necesitar saber inglés (P13).

\section{Influencia de los medios de comunicación}

P13 atribuye la desmotivación a causas procedentes de los medios de comunicación. La familiarización con profesionales que ocupan puestos relevantes, o que trabajan en el mundo de la información, del deporte, del espectáculo e incluso de la política, es decir profesionales de la palabra (P41), que no dominan idiomas, son un ejemplo pernicioso para la motivación del estudiante: Hacen un ridículo espantoso cada vez que tienen que hablar en inglés y se quedan 'tan panchos' (P41). Así mismo, el encuestado alude a la ausencia de idiomas en el entorno cotidiano, en el que se destacan y veneran los estereotipos locales e impera la inexistencia de perspectivas internacionales (P41). Otro profesor relata: (...) la imagen que los medios de comunicación ofrecen del inglés y las culturas a él vinculadas (P17).

Otro docente manifiesta: El alumnado ha cambiado, sólo les interesa hacer aquello que les genere dinero en un futuro y el francés no les va a solucionar sus vicios. Creo que la culpa de esto se debe a los medios de comunicación (P53).

\section{Espíritu localista}

Dos enseñantes se refieren a la creación de estereotipos de la "otra" realidad cultural, como causa de generación de desmotivación de los alumnos: 
(...) el conocimiento muy superficial, construido mayormente de estereotipos, del otro "en Inglaterra no comen", "todos los ingleses son unos hooligans", "si los ingleses no saben español, ¿por qué nosotros tenemos que hablar inglés?", "los extranjeros vienen a España porque aquí se vive mejor que en ninguna otra parte del mundo"...) y un bagaje cultural generalmente bajo (P17).

\section{Causas académicas}

Carácter obligatorio de la enseñanza-aprendizaje hasta los 16 años y obligatoriedad de cursar un idioma extranjero.

Encontramos algunos relatos en los que se alude al carácter obligatorio del aprendizaje de idiomas. La imposición de su estudio desencadena sentimientos de falta de responsabilidad y no toman conciencia de la utilidad de lo que aprenden (P5). Un profesor de Secundaria lo resume así: es fundamental tener alumnos que quieran trabajar y que vean en la segunda lengua extranjera una cierta obligatoriedad no que piensen que es "algo que lo escojo si quiero" (P28).

Otro docente expresa que no existen diferencias en cuanto a la motivación o desmotivación del alumno con respecto al aprendizaje de otras materias. Su origen se debe a que la enseñanza es obligatoria hasta los 16 años:

La lengua extranjera forma parte, como el resto de las asignaturas, del currículo y por lo tanto tiene carácter obligatorio hasta los 16 años. Muchos/as alumnos/as carecen de interés tanto por ésta como por el resto de materias (P27).

Otro profesor reprocha la promoción automática de los cursos, puesto que (...)

aunque no hagan nada, ni sepan nada, ni aprendan nada, aún así van a promocionar al siguiente curso, con lo cual ¿para qué esforzarse? (P9).

Curriculum sobrecargado de asignaturas y falta de claridad en los objetivos

Cuatro docentes, afirman que el hecho de (...) que los alumnos tengan tantas asignaturas por año solamente trae consigo agobios, disgustos y mucho cansancio por su parte (P29). Otro profesor (P48), del ámbito universitario, señala que el currículum sobrecargado de contenidos supone para los alumnos la presión de un calendario académico muy apretado para la realización de los exámenes.

Otra de las causas de carácter metodológico que genera el descenso de la autoconfianza y de la autoestima es la falta de claridad de los objetivos planteados por el profesor. El desconocimiento del alumno de cómo poner en práctica determinadas estrategias que le permitan dirigir su esfuerzo hacia la consecución de las metas, así como el no saber cómo y cuándo aplicarlas suele conducirles a la desmotivación y, en consecuencia, a la falta de esfuerzo en la dirección adecuada. 


\section{DISCUSIÓN DE LOS RESULTADOS Y CONCLUSIONES}

Estos datos avalan que el contexto familiar, social y cultural del que procede el alumno es una de las causas principales de su motivación en el proceso de enseñanza-aprendizaje. Se aprecia un aumento de la motivación en aquellos cuyas condiciones económicas y socioculturales son favorables, en los estudiantes cuyas familias muestran apoyo afectivo e interés por la evolución de su proceso de aprendizaje. Por el contrario, relacionan la desmotivación y el fracaso con la falta de alicientes, estímulos e indiferencia familiar y del entorno en el que conviven. Por lo general, son familias desestructuradas, con escasos recursos económicos y con bajo nivel cultural y en consecuencia con un limitado nivel lingüístico de la lengua materna. Estos resultados coinciden con numerosas investigaciones (Gonzalez-Pienda et al., 2002; López Larrosa, 2002; Alonso Tapia, 2005) que apoyan la tesis de la influencia determinante del ambiente familiar en el rendimiento académico de los alumnos y en su motivación hacia el proceso de enseñanza-aprendizaje.

En virtud de la respuestas podemos definir como generadores de la motivación, la presencia de libros, la asiduidad de viajes al extranjero, el interés personal y familiar por el conocimiento de la nueva cultura, la atención que los padres prestan a los hijos, la valoración que la familia realiza del individuo que domina una lengua extranjera, su optimismo con las expectativas de éxito de los hijos, el interés por sus actividades escolares, el contacto frecuente con los profesores de idiomas y con el centro educativo. Sin embargo, llama la atención que algunos docentes aseguren un descenso general de la motivación en los alumnos de la etapa de Secundaria, cuyas causas son independientes de la posición económica-social familiar. Quizás se deba, por un lado, como destacan Madrid et al. (1993), a que la influencia paterna decrece como generador motivacional conforme avanzan los cursos y adquiere mayor relevancia el contexto de aprendizaje. Por otro, a que los intereses de los alumnos cambian con la edad. En lo que la mayoría de los profesores coincide es en que la motivación es mayor en edades infantiles. A este respecto, aunque encontramos alguna opinión discrepante, el conjunto de encuestados confluye en la existencia de tres etapas diferentes:

Edad infantil. Unos docentes consideran que en edades tempranas los aprendices están más motivados, son más desinhibidos, alimentan su curiosidad indagando en temas nuevos, no se sienten inseguros cuando hacen uso de su competencia oral con el idioma, presentan actitudes más positivas hacia el aprendizaje y su autoestima no se encuentra amenazada por los errores cometidos. Sin duda, de acuerdo con Guirora et al., (1972), el ego-lingüístico se encuentra íntimamente relacionado con la edad. A medida que ésta avanza el ego-lingüístico 
va perdiendo permeabilidad y plasticidad, motivo por el que el niño se encuentra en condiciones lingüísticas más receptivas y por el que el adulto se muestra más rígido, lo cual limita sus posibilidades de aprendizaje.

La observación de los profesores en el aula revela que en estas edades existe una percepción de la lengua como algo sencillo, les oigo que el idioma es la asignatura más fácil comenta una informante (P58), explicación que los docentes atribuyen al escaso uso de las aclaraciones gramaticales y al empleo de estrategias y actividades con un gran componente lúdico como canciones, bailes, etc. Los estudios llevados a cabo por Burstall (1975) demostraron que los alumnos de ocho años mostraban actitudes más positivas que los mayores pero esto no se reflejaba en mejores habilidades orales. Las investigaciones realizadas por Tragant y Muñoz Lahoz (2000) con estudiantes de EP, EGB, ESO, BUP y COU demostraron que comenzar a los ocho o a los once años no afecta la actitud global hacia la lengua extranjera de manera significativa (p.e.: 2000, 92). Cabe destacar en su investigación que las horas de clase recibidas producen un efecto más positivo en su motivación y en la actitud que la edad. Los resultados obtenidos de la etapa de BUP y COU la motivación es mayor, explicación que puede tener su origen en la existencia de filtros selectivos anteriores porque estos cursos forman parte de la enseñanza secundaria postobligatoria (p.e.: Tragant y Muñoz Lahoz, 2000, 93).

Edad de la pubertad: En varios relatos se describe la falta de interés, la apatía, la indiferencia de los aprendices, e incluso algunos especifican haber notado amotivación especialmente en las etapas de la ESO. Los alumnos más motivados se encuentran en Primero y Segundo de la ESO; sin embargo, disminuye progresivamente su motivación a medida que cursan estudios superiores. La causa que motiva a estos estudiantes, especialmente de Bachillerato, hacia el aprendizaje de una lengua extranjera es la expectativa profesional. Estudios llevados a cabo por psicolingüistas y psicoanalistas han demostrado que cuando se aprende una segunda lengua tras la pubertad ambas lenguas difieren en su impacto emocional, convirtiéndose la primera en la de mayor impacto personal y la segunda en la lengua que tiene menos carga emotiva (p.e.: Pavlenko, 2002: 47). Ésta puede ser la razón por la que, por ejemplo, algunos exiliados prefieren escribir en la segunda lengua al no tener connotaciones emocionales vinculadas a la política.

Edad de la madurez: Respecto a esta etapa encontramos dos opiniones que divergen con las del colectivo de profesores. Ambos, docentes de la etapa educativa de Bachillerato, afirman que la motivación resulta más efectiva cuando los alumnos adquieren una mayor madurez intelectual y seguridad en sí mismos. Por el contrario, mayoritariamente consideran que cuando la exigencia en el idioma es mayor, como ocurre en Bachillerato, la motivación disminuye, pues se requiere 
del estudiante un incremento del esfuerzo para alcanzar las metas. Al ser esta edad susceptible de cambios emocionales e intereses personales y en la que surgen conflictos que diluyen el interés por el aprendizaje y la dedicación al estudio, es muy difícil mantener la motivación, especialmente en aquellos alumnos que no han pensado continuar con su formación en la universidad. Otros motivos pueden ser, recordando a Guirora et al. (1972) y su teoría a cerca del ego-lingüístico, que el adulto al ser más autocrítico sufra una mayor frustración.

En consecuencia de lo expresado, a una edad adulta se debería ser menos autocrítico, con mayor sentido del humor ante los errores y menos preocupado por el papel social. Además, podríamos suponer que el interés por otra cultura se incremente con la edad. Sin embargo, sabemos que el aprendizaje de un idioma no supone únicamente un medio para lograr la comunicación con los hablantes de otra realidad cultural. El individuo se vale del lenguaje para configurar su propia personalidad, proyectando su "yo" en la lengua, lo que se ha denominado como ego-lingüistico (Guirora et al., 1972). La palabra se convierte, por tanto, en definitoria de la personalidad del individuo, erigiéndose el lenguaje como el elemento de representación de la propia individualidad. Una de las expresiones comúnmente conocida, y que en nuestra opinión, puede aclarar este planteamiento es, por ejemplo, los niños son como esponjas, es decir, absorben, procesan y utilizan todo lo que oyen y aprenden. Su ego-lingüístico es aún plástico, permeable, flexible. Podemos deducir de ello que el niño, puesto que aún no posee la seguridad y confirmación que le confiere el primer idioma, se encuentra en unas condiciones lingüísticas más receptivas. Por el contrario el adulto, seguro en el empleo de su primer idioma, se muestra poco inclinado a hacer uso de un modo nuevo de "discurso"; su actitud hacia la nueva forma de expresión se muestra más rígida, llegando incluso, en ocasiones, a rechazarla, con lo que el uso del filtro afectivo es mucho mayor (Stern, 1983 y Coste, 1984). Obviamente, este hecho limitará sus posibilidades de aprendizaje. Por consiguiente, opinamos que el adulto debería adaptarse al nuevo estatus y prepararse sin miedo a la adquisición y/o confluencia de las dos realidades lingüísticas, la de su lengua materna y la de la lengua meta.

Otros motivos personales que los docentes relacionan con la motivación son la autoestima y el sentimiento de seguridad del sujeto. Algunos docentes conocen bien sus efectos positivos y con la intención de fomentar en el alumno el "sentimiento de valía", emplean estrategias como la de reconocer públicamente la capacidad intelectual del aprendiz, elogiar y congratular sus actuaciones y restar importancia a la comisión de errores. No olvidemos que cualquier evento que sucede en el aula influye de forma determinante en la motivación del sujeto hacia el aprendizaje. 
Los profesores coinciden en afirmar que la raíz de los problemas afectivos que genera la desmotivación en el aprendizaje de un idioma radica, principalmente, en el miedo del alumno al fracaso, en su autoimagen deficiente y en el sentimiento de inadecuación. Los resultados de la investigación coinciden con los realizados por Bernau (p.e.: 2001, 10) al confirmarse que una autoestima positiva asegura que el proceso de aprendizaje no se detenga, mientras que si el alumno adolece de una baja autoestima no logrará alcanzar un nivel adecuado de aprendizaje. La autora afirma que la autoestima varía según el tipo de tareas a las que se enfrenta una persona y la relaciona con la autoevaluación de una tarea (p.e.: 2001, 83). Si el alumno adquiere buenos resultados, su autoestima es alta; por el contrario, si su autoevaluación es negativa, ésta descenderá.

De ello se deriva que los efectos de una autoevaluación positiva median en la autoestima y motivación, al igual que, como apuntamos anteriormente, la estima positiva de los estudiantes depende de la consideración que las personas significativas tienen de ellos, los padres, profesores y compañeros. Especialmente los profesores de Primaria destacan la necesidad de mostrar cariño, aprecio y mantener una relación respetuosa para fomentar la autoestima. Una retroalimentación continua de comentarios críticos en el aula puede generar sentimientos negativos en sus experiencias de aprendizaje. Sin embargo, no todos los docentes parten de que todos los alumnos posean las aptitudes y capacidades intelectuales necesarias para el aprendizaje de un idioma. Por ello, nos cuestionamos la dificultad del aprendiz para salvaguardar su autoestima si, ya desde edades infantiles, el docente duda de sus capacidades y aptitudes. A nuestro juicio, el docente tiene que considerar que su pensamiento va a influir en los propios alumnos, pues como postula Cambra Giné ((p.e.: 2003, 272), en el aula existe una influencia entre los profesores y los alumnos a nivel de representaciones y pensamiento, es decir, la acción educativa no se limita exclusivamente a la transmisión de saberes, los profesores transmiten sus valores, creencias, representaciones ${ }^{5}$, etc.

Otros motivos, fuente de motivación, citados por los encuestados y que coinciden con las investigaciones de autores como Gardner y Lambert (1985) son

${ }^{5}$ En palabras del autor: L'action éducative ne se limite pas à la transmission de savoirs: des valeurs sont aussi transmises à travers les comportements et les règles du jeu établies tacitement ou explicitement. Les consignes, sollicitations, reprises, réparations, évaluations modèlent constamment le comportement verbal des apprenants et ont une influence certaine dans la formation des systèmes de RCS [représentations, croyances, savoirs] des apprenants. (p.e:Cambra Giné, 2003, 272). 
el ánimo de identificarse con la vida cultural y social de los hablantes de la lengua meta, de entablar amistades, conocer las modas y costumbres e integrarse en una nueva comunidad. Algunos, como Dörnyei (1994), opinan que para aprender una lengua es necesaria una orientación integradora, estrechamente relacionada y anclada en la personalidad del sujeto. En las encuestas hemos encontrado opiniones que confirman esta tesis e incluso que priman la influencia de este tipo de motivación. Otros docentes van más lejos al afirmar que si el individuo no se identifica con la comunidad de hablantes, optará por alejarse de ella. Si es así, somos conscientes de la difícil tarea del profesor para que en el contexto del aula sea posible una identificación con el "otro", superando dificultades como las de proporcionar suficientes datos sobre las costumbres de la cultura extranjera con el riesgo de poder generar una visión bastante estereotipada de la otra cultura, o la ausencia de contacto de los alumnos con miembros de la cultura de la lengua meta. Para superarlas los docentes proponen la realización de intercambios, viajes y estancias en el país de lengua meta.

En las encuestas encontramos varias referencias a la actitud del profesor en el aula como fuente generadora de motivación. Como afirma Martínez Aguado (2001) el docente puede modificar los intereses, las necesidades e incluso las habilidades del individuo. Los profesores destacan la influencia de su estilo personal de enseñanza, también señalada por Wasna (1972), sus estrategias para promover la interacción y su empeño por fomentar la empatía entre él y el grupo de alumnos. Para el desarrollo de la empatía los encuestados subrayan positivamente su carácter extravertido, en otras palabras, la tendencia de un individuo a ser más abierto y estar pendiente del entorno que le rodea y de las personas con las que convive. Partiendo de este concepto, podemos comprender que el docente extravertido ofrezca al estudiante mayor seguridad, confianza y bienestar en el aula. Otro de los rasgos del docente para el desarrollo de la empatía en el aula es su sentido del humor. En las encuestas se especifica que el humor y la risa mejoran el proceso de aprendizaje. Sin embargo, nos cuestionamos si este rasgo del carácter del profesor es común a todos los individuos, si el humor es una característica intrínseca a la personalidad del docente o, por el contrario, cultural. Si resulta lo segundo, sería interesante investigar en técnicas que lo potencien.

En relación con la actitud del docente algunos encuestados enfatizan su ilusión por transmitir las referencias culturales, su empeño por el desarrollo de interacción profesor-alumno y su forma de escuchar. En definitiva, estos informantes describen el papel del profesor como el de guía del aprendizaje, con una buena dosis de imaginación, como sostiene Arnold (2000). Además, hemos observado que estos docentes promueven la colaboración de los alumnos en el diseño de las clases y 
actividades, concediéndoles un gran protagonismo. Así mismo, los profesores consideran la comisión de los errores como parte del aprendizaje y procuran que el alumno no los conciba como un castigo.

A continuación nos ocuparemos de las causas académicas destacadas por los encuestados.

En virtud de los hechos mencionados el docente ha de tener en cuenta las atribuciones que los estudiantes realizan de sus fracasos anteriores en el proceso de aprendizaje y la necesidad de éxito como indicamos anteriormente. Estos aspectos de la motivación, también aportados por Dörnyei (1994), son inherentes al concepto global de la motivación. Navarro, Luzón y Villanueva (1997) concluyen que los comportamientos de aprendizaje, estrategias, hábitos, etc. adquiridos durante la formación tendrán una influencia decisiva en los procesos posteriores.

Otras causas de carácter práctico, están asociadas a intereses de tipo pragmático como el logro de mejoras profesionales o la obtención de un buen expediente académico, lo cual llevó a definir la motivación como instrumental (Gardner y Lambert, 1972).

En relación al contexto de aprendizaje, las encuestas confirman la relevancia de los factores propios del aula y la dinámica de grupo, un factor relacionado con el tamaño del grupo. Sin duda los centros educativos deberían duplicar sus esfuerzos para la creación de grupos pequeños, puesto que las encuestas confirman que cuanto más pequeño es éste, mayor es la motivación.

En un contexto de centro favorable, donde la propia institución concede a la lengua extranjera especial relevancia existe un mayor aumento de la motivación. Este es el caso, por ejemplo, de los institutos en los que se imparte Bachillerato Internacional o enseñanzas bilingües. Destacamos una encuesta (P21) en la que un profesor se lamenta de la política de los centros en los que únicamente se permite el estudio de una segunda lengua extranjera a los alumnos que obtienen resultados positivos en las asignaturas instrumentales. Ello genera el rechazo hacia el idioma de los alumnos que cursan otra lengua como obligatoria.

Entre las causas metodológicas aducidas por los informantes destacan especialmente el enfoque comunicativo; una enseñanza en la que se prime el trabajo colaborativo y en la que atienda a las necesidades individuales. Sin duda, la tarea del docente es de enorme trascendencia puesto que, además, tendrá que valorar el grado de dificultad de las tareas. Ambas causas han de estar en consonancia con el nivel del alumno, ni por encima ni por debajo de sus conocimientos porque pueden resultarles incomprensibles y/o aburridas. Si el alumno tiene que estudiar más de lo debido será también causa de desmotivación. Además, estos docentes, en nuestra opinión, entienden que el aprendizaje debe ser 
inductivo, enraizado en la práctica, al igual que se aprende el idioma materno, sin convertirlo en una tarea consciente a través de la abstracción de reglas gramaticales. No obstante, no podemos olvidar aquellas encuestas que abogaban por la práctica de ejercicios gramaticales y de memorización. Sin embargo, y de manera general, sus pensamientos nos recuerdan en algunos aspectos al método directo en el sentido de que para los docentes la motivación, y en consecuencia el aprendizaje, se logra por medio de la interacción comunicativa profesor-alumno, centrada en el desarrollo de las destrezas orales y no escritas, así como el recurso a materiales con dibujos y el lenguaje corporal que facilitan la comprensión de significados.

A continuación abordamos las causas mencionadas por los encuestados implicadas en la desmotivación de los alumnos.

De las continuas alusiones a la realización de ejercicios gramaticales y al empeño del docente por corregir los errores del alumno podemos concluir, por un lado, la necesidad de realizar ambas tareas, y por otro la conveniencia de cambiar las formas de llevarlas a la práctica. Las encuestas son un testimonio de que ambas actividades, cuando se realizan en exceso, generan sentimientos negativos como ridículo y miedo a intervenir en clase.

Creemos, además, que las Administraciones educativas deberían apoyar a los centros de enseñanza, especialmente a aquellos cuyos alumnos procedan de colectivos sociales más desfavorecidos, entre los que destacan las zonas rurales en las que las familias no valoran la adquisición de un idioma — no le ven proyección para su futuro profesional, suelen dedicar menos tiempo a sus hijos en las tareas escolares y no se interesan por viajar o conocer otras realidades-. Ese refuerzo puede darse, por ejemplo, con profesores de apoyo o con un número reducido de alumnos por clase.

Así mismo, debería exigirse a los profesionales de la información e incluso representantes políticos, personajes públicos, del espectáculo etc., el manejo adecuado de un idioma extranjero. Los medios de información deberían, también, colaborar en el cambio de mentalidad respecto a los hablantes de la lengua extranjera, evitar el espíritu localista y las representaciones estereotipadas del país de la lengua meta.

En relación con las causas académicas, es notoria la frecuencia con la que los profesores se refieren a la obligatoriedad de la enseñanza hasta los 16 años y a la promoción automática de curso con asignaturas suspensas en la ESO. Ningún profesor pone en duda que la influencia de estos alumnos es perjudicial para los estudiantes motivados. Algunas investigaciones han demostrado que el carácter de optatividad y obligatoriedad de una materia determina la calidad de la motivación 
del alumno; si su estudio es optativo los alumnos presentan actitudes más positivas y una motivación más alta (Martínez Aguado, 2001; Muñoz, 2000). En palabras de Alonso Tapia: Cuando la decisión de hacer algo surge de uno mismo, el chico o chica experimentan que son los que controlan su conducta, que no son una "marioneta" actuando porque lo quieren los demás y al servicio de intereses ajenos (p.e.: 2005, 27).

Otras alusiones negativas, procedentes en su mayoría de docentes universitarios, se refieren a los planes de estudio. Únicamente un profesor de Secundaria alude negativamente al número excesivo de asignaturas en el currículo del alumno, que impide disponer de tiempo suficiente para profundizar en las materias y que generan tensiones y confusión.

\section{REFLEXIÓN FINAL}

Para concluir, y a modo de propuestas pedagógicas, exponemos seguidamente algunas reflexiones surgidas de nuestra investigación.

En primer lugar, creemos necesario dejar constancia de las críticas que prácticamente la totalidad de los informantes realizan de la enseñanza de lenguas extranjeras centrada en metodologías exclusivamente memorísticas, sin contextualizar y cuyo objetivo era aprender los contenidos correspondientes. Especialmente lamentan que la práctica de procesos de comunicación en el aula y la transferencia de esa práctica no se dirige a situaciones reales de comunicación. Abogamos, por tanto, por una metodología que permita desarrollar actividades de comunicación y de aprendizaje en contextos significativos y estimulantes para los alumnos.

En segundo término, nos ha llamado poderosamente la atención que un número apreciable de docentes no se haya cuestionado la existencia de las emociones y sus implicaciones en el proceso de enseñanza-aprendizaje; que no haya estimado que el profesor, al estar en una situación grupal, su tono de voz, su humor, su ritmo de habla, sus gestos, su proximidad o distancia física con el alumno, etc. influyen en los procesos cognitivos de los estudiantes y en sus actitudes hacia el aprendizaje de la nueva lengua-cultura. Por ello, sugerimos completar la formación psicopedagógica de los profesores con el propósito de adquirir conocimientos $\mathrm{y}$ destrezas que permitan el manejo eficaz de las emociones en el aula de idiomas.

En nuestra opinión, no le corresponde al docente tratar los problemas psíquicos de los alumnos, sin embargo, si adquiere un conocimiento empático, desde el vínculo didáctico que puede establecer con ellos, puede modificar comportamientos y generar un clima agradable que inste a la actitud positiva hacia el proceso de aprendizaje. Los profesores de lenguas extranjeras de todos los 
ámbitos educativos deben esforzarse por crear una atmósfera que facilite los procesos cognitivos, que promueva respuestas emotivo-afectivas positivas y el desarrollo de las destrezas comunicativas.

Creemos, en consonancia con los últimos estudios sobre los métodos de enseñanza, que para obtener éxito el docente debe modificar la perspectiva clásica desde la que se plantea su ejercicio profesional y abandonar el papel de protagonista, de mero trasmisor de conocimientos. Ha de enfocar la enseñanza hacia los alumnos y reconocerlos como los auténticos protagonistas, en consecuencia, promover los cauces necesarios para facilitarles el éxito en el aprendizaje. El reconocimiento de la individualidad de los aprendices supone que se debe tener en cuenta la importancia que tienen un gran número de variables que interactúan en el proceso de aprendizaje como, por ejemplo, la actitud ante la lengua, la influencia del contexto (los padres, la relación con el profesor-grupo de alumnos), la motivación, y las consecuencias que todas ellas tienen en la generación en las aulas de afectos positivos o negativos que potencian o lastran el aprendizaje.

Otro eje fundamental en el pensamiento de los docentes determina que el aprendizaje de una segunda lengua posee ciertas connotaciones especiales que lo hacen diferente al de otras materias. Por un lado, aprender otra lengua requiere la socialización con otras personas; sin embargo, no todos los alumnos reaccionan de la misma forma en situaciones comunicativas. Además, las aulas de idiomas suelen diseñarse en torno a situaciones grupales en las que el alumno se ve obligado a participar, pudiendo despertar emociones negativas o temores antiguos. Por otra parte, la lengua está relacionada con la identidad del individuo, es parte de nuestro ser social y el vehículo de trasmisión de la propia identidad a los otros. Para unos aprendices la nueva lengua puede ser el medio que viabilice la expresión de sentimientos que la lengua materna silencia. En cambio, para otros los convierte en seres más vulnerables al tener que expresar sentimientos cuando su dominio de la lengua es todavía precario.

Uno de los factores que contribuyen más poderosamente a fomentar, mantener o generar la motivación y las emociones positivas hacia el proceso de enseñanzaaprendizaje de un idioma es el contacto directo de los alumnos y de los profesores con el país de la lengua extranjera. Por ello, es necesario que ambos colectivos realicen estancias formativas en el extranjero y que la Administración Educativa y los centros fomenten el número de becas, los intercambios escolares, las actividades culturales relacionadas con la lengua-cultura extranjera y que se incremente la participación en los diferentes programas de lenguas: Erasmus, Sócrates, etc. Así pues, apoyamos, haciéndonos eco de la demanda prácticamente 
generalizada entre los docentes de Primaria y Secundaria, la conveniencia de facilitar a los profesores de idiomas estancias formativas en el país de la lengua meta durante el curso escolar, pues redundarían directamente en el enriquecimiento de su labor docente.

\section{REFERENCIAS BIBLIOGRÁFICAS}

ALONSO TAPIA, J. (2005): Motivar en la escuela, motivar en la familia, Madrid, Morata.

AMMES, C. y AMES, R. E. (Eds.) (1984). Research on Motivation in Education. Vol.I, Student Motivation, Londres. Academic Press.

AMMES, C. y AMES, R. E. (Eds.) (1985): Research on Motivation in Education, Vol.II, The Classroom Milieu, Londres, Academic Press.

AMMES, C. y AMES, R. E. (Eds.) (1989): Research on Motivation in Education, Vol.III. Goals and Cognitions. Milieu, Londres, Academic Press.

ARNOLD, J. (2000): La dimensión afectiva en el aprendizaje de idiomas, Madrid, Cambridge University Press.

ATIENZA MERINO, J. L. (Dir.), BLANCO HÖLSCHER, M., FÁÑEZ PÉREZ, B., IGLESIAS CASAL, I., LÓPEZ TÉLLEZ, G., LÓPEZ VÁZQUEZ, M. A. y MARTÍNEZ GARCÍA, J. A. (2005): ¿Cómo se ven? ¿Cómo nos ven? Atrapados en los estereotipos. Una investigación sobre las representaciones culturales de estudiantes extranjeros de la Universidad de Oviedo, Oviedo, Servicio de Publicaciones de la Universidad de Oviedo.

BERNAUS, M. y ESCOBAR, C. (2001): "El aprendizaje de lenguas extranjeras en medio escolar", en Didáctica de las lenguas extranjeras en la Educación Secundaria Obligatoria, NUSSBAUM, L. y BERNAUS, M. (Eds.), Madrid, Editorial Síntesis, 39-77).

BOLÍVAR , A., DOMINGO. J. y MANUEL F. (2001): La investigación biográfico-narrativa en educación. Enfoque y metodología, Madrid, Editorial la Muralla, S.A.

BURSTALL, C. (1975): "French in the Primary School: The British Experiment", The Canadian Modern Language Review, 31, (5), 388-402.

CAMBRA GINÉ, M. (2003): Une approche ethnographique de la classe de langue, Paris, Didier.

COLÁS BRAVO, M. P. y BUENDÍA EISMAN, L. (1994): Investigación educativa ( $2^{\mathrm{a}}$ ed.), Sevilla, Alfar.

COSTE, D. (1984): "L'interaction en classe de langue et le development de l'apprenent comme sujet psychologique et acteur social", en Vers un Cadre 
Elargi pour la Définition des Objectifs de l'Apprentissage des Langues, Strasbourg, Conseil de L'Europe.

DÖRNYEI, Z. (1994): "Motivation and Motivating in the Foreign Language Classroom“, Modern Language Journal, 78, 273-84.

DÖRNYEI, Z. (1998): "Survey Article: Motivation in Second and Foreign Language Learning", Language Teaching, 31, 117-135.

GARDNER, R. y LAMBERT W. E. (1959): "Motivational Variables in SecondLanguage Acquisition", Canadian Journal of Psychologie, 13 (4), 266-272.

GIL, X., GARCÍA, E. y RODRÍGUEZ, G. (1995): "Anàlisi de dades en la investigació etnogràfica", Temps d'Educació, 14, 61-82.

GONZALEZ-PIENDA, J. A., NÚNEZ, J. C., ÁVAREZ, L., GÓNZALEZPUMARIEGA, S., ROCES, C., GONZÁLEZ, P., MUÑIZ, R., BERNARDO, A. (2002):'Inducción parental a la autorregulación, autoconcepto y rendimiento académico", Psicotema, 14 (4), 853-860

GUIORA, A. (1984): "The Dialect of Language Acquisition", Language Learning, $35,3-12$.

GUIRORA, A., BRANNON, R. y DULL, C. (1972): "Empathy and Second Language Learning", Language Learning, 22, 111-130.

LECOMPTE, M. (2000): “Analyzing Qualitative data", Theory Into Practice, 39 (3), 146-154.

LÓPEZ LARROSA, S. (2002): “El FACES II en la evaluación de la cohesión y la adaptabilidad familiar", Psicotehema, 14, 159-166.

MADRID, D., GALLEGO, J. A., RODRÍGUEZ, J., URBANO, B., FERNÁNDEZ, J., MANRIQUE, I., HIDALGO, E. y LEYVA, C. (1994): "Motivación, rendimiento y personalidad en el aula de idioma", en Actas de las IX Jornadas Pedagógicas para la enseñanza de inglés, Granada, GRETA, 198-214.

MANOLOPOULOU-SERGI, E. (2004): "Motivation Within the Information Processing Model of Foreign Language Learning", System, 32, 427-441.

MARTÍNEZ AGUADO, J. D. (2001): "La activación y mantenimiento de la motivación durante el proceso de enseñanza-aprendizaje de una lengua extranjera”, en Didáctica (Lengua y Literatura), 13, 235-261.

MUÑOZ JUSTICIA, J. Análisis cualitativo de datos textuales con Atlas.ti 5, versión 3.03 [en línea]. Disponible en Web: http://www.fcp.uncu.edu.ar/upload/Atlas5_manual.pdf. [Consulta: 17 de abril de 2006].

MUÑOZ, C. (2000): Segundas Lenguas. Adquisición en el aula, Barcelona, Ariel Lingüística S.A. 
NAVARRO, I., LUZÓN, Mª J. y VILLANUEVA, Ma L (1997): "Estudio de las representaciones lingüísticas de los estudiantes de la UJI: cultura de enseñanza vs. cultura de aprendizaje", en Los estilos de aprendizaje de lenguas. Un estudio sobre las representaciones culturales y las interacciones de enseñanzaaprendizaje, VILLANUEVA, M. L. y NAVARRO, I. (Eds.), Castelló, Publicaciones de la Universitat Jaume, 103-154.

PAVLENKO, A. (2002): "Bilingualism and Emotions", Multilingua, 21, 45-78.

PAVLENKO, A. (2002): "Bilingualism and Emotions", Multilingua, 21, 45-78.

STERN, H. H. (1983), Fundamental Concepts of Language Teaching, Oxford, Oxford University Press.

TRAGANT, E. y MUÑOZ C. (2000): "La motivación y su relación con la edad en un contexto escolar de aprendizaje de una lengua extranjera", en Segundas Lenguas. Adquisición en el aula, MUÑOZ, C. (Ed), Barcelona, Editorial Ariel, 81-105.

WASNA M. (1972): Motivation, Intelligenz und Lernerfolg, München, KöselVerlag GmbH \& Co.

WILLIAMS, M. y BURDEN, R. L. (1999): Psicología para profesores de idiomas. Enfoque del constructivismo social, Madrid, Cambridge University Press. 